\title{
Impact of Continuous Positive Airway Pressure Therapy on Atrial Electromechanical Delay and P-Wave Dispersion in Patients with Obstructive Sleep Apnea
}

\author{
Pınar Türker Bayır, M.D., * Burcu Demirkan, M.D.,† Ömer Bayır, M.D. $\neq$ \\ Serkan Duyuler, M.D.,§ Hikmet Firat, M.D., đÜmit Güray, M.D.,* \\ Yeşim Güray, M.D. $\dagger$ and Emel Çadallı Tatar, M.D.‡
}

From the *Department of Cardiology, Ankara Numune Education and Research Hospital, Ankara, Turkey; $\dagger$ Department of Cardiology, Turkiye Yuksek Ihtisas Hospital, Ankara, Turkey; $\$$ Department of Otolaryngology Head and Neck Surgery, Ministry of Health Dışkapı Yildırtm Beyazıt Research and Educational Hospital, Ankara, Turkey; §Department of Cardiology, Hakkari State Hospital, Hakkari, Turkey; and $\$$ Department of Chest Medicine and Sleep Disorders Center, Ministry of Health Dışkapı Yildırım Beyazıt Research and Educational Hospital, Ankara, Turkey

Background: Obstructive sleep apnea (OSA) has been shown to be associated with atrial fibrillation (AF). Prolongation of inter- and intraatrial conduction times during sinus rhythm has also been shown to be related to AF generation. Nasal continuous positive airway pressure (CPAP) is an effective treatment modality of OSA.

Methods: Twenty-four OSA patients diagnosed through polysomnography and 18 controls were included in the study. The basal inter- and intraatrial electromechanic delays prior to onset of the therapy were measured using tissue Doppler imaging. P-wave dispersion $(\mathrm{Pd})$ was calculated on the basis of 12-lead electrocardiography. Same measurements were performed in OSA patients 6 months after the initiation of the therapy.

Results: Interatrial $(39.2 \pm 8$ vs. $21.1 \pm 2.8 \mathrm{~ms}, \mathrm{P}<0.001)$, left intraatrial $(20.5 \pm 7.2$ vs. $11.1 \pm 2 \mathrm{~ms}, \mathrm{P}=0.003)$, and right intraatrial electromechanical delays $(20.7 \pm 11 \mathrm{vs} .10 \pm$ $2.6 \mathrm{~ms}, \mathrm{P}<0.001$ ) prior to CPAP therapy were found to be significantly greater in OSA group as compared with the controls. Pd was also greater in the OSA group as compared with the controls $(44 \pm 7$ vs. $28.5 \pm 4$ ms, $P<0.001)$. However, significant improvement has been noted after 6 months of CPAP therapy in interatrial $(P<0.0001)$, left intraatrial $(P=0.002)$, and right intraatrial electromechanical delays $(\mathrm{P}<0.0001)$ as well as in $\mathrm{Pd}(\mathrm{P}<0.0001)$ as compared to baseline values in patients with OSA.

Conclusion: Our findings suggested that CPAP therapy provides more homogenous conduction through atria in patients with OSA. This effect may translate into decreased risk for AF associated with OSA.

Ann Noninvasive Electrocardiol 2014;19(3):226-233

obstructive sleep apnea; continuous positive airway pressure; atrial electromechanical delay;

P-wave dispersion

Obstructive sleep apnea (OSA) is the most common sleep disorder characterized by repetitive complete or partial collapse of the upper airway and arousals from sleep because of strenuous breath activity. ${ }^{1}$ With the incidence of $5-15 \%$, OSA is known to be related to cardiac failure (left and right ventricle dysfunction), hypertension, coronary artery disease, pulmonary hypertension, and cardiac arrhythmias. ${ }^{2,3}$

Atrial fibrillation $(\mathrm{AF})$ is the most common sustained arrhythmia encountered in clinical practice, also the major cause of being hospitalized

Address for correspondence: Pinar Türker Bayır, M.D., Department of Cardiology, Ankara Numune Education and Research Training Hospital, Talatpaşa Bulv.06100, Sihhyye, Ankara, Turkey. Fax: + 9031231034 60; E-mail: turkerpinar1982@hotmail.com 
and an important reason for morbidity and mortality. ${ }^{4,5}$ Previous studies have found a close link between AF and OSA. ${ }^{6}$ Electrophysiological and electromechanical abnormalities resulting with inter- and intraatrial conduction delays increase the risk of $\mathrm{AF}^{7}$ Prolonged inter- and intraatrial conduction times can be demonstrated by tissue Doppler imaging (TDI) and by electrocardiographic parameters, such as maximal P-wave duration (Pmax) and P-wave dispersion (Pd). ${ }^{8,9}$ These parameters have been found to related to increase $\mathrm{AF}$ risk in various clinical conditions such as hypertension, diastolic dysfunction and mitral stenosis. ${ }^{10-12}$ In addition, Yagmur et al. have shown prolonged inter- and intraatrial conduction times in patients with moderate-to-severe OSA as compared to controls. ${ }^{13}$ Cagirci et al. have also demonstrated that the magnitude of prolongation in atrial electromechanic delay and increase in $\mathrm{Pd}$ is proportional to disease severity in patients with OSA. ${ }^{14}$ Nasal continuous positive airway pressure (CPAP) is an efficient and widely used method for treatment of patients with moderate-to-severe OSA. ${ }^{15,16}$ It has been documented that CPAP both decreases the OSA-related symptoms and cardiovascular mortality. ${ }^{17}$ However, to date, the effect of CPAP therapy on the electrocardiography (ECG) and echocardiographic parameters of the susceptibility of AF is unknown. The objective of this study is to evaluate the impact of CPAP therapy on atrial conduction time which can be measured by TDI therapy and electrocardiographic parameters such as Pmax and Pd.

\section{METHODS}

\section{Study Population}

Patients and control groups that were subjected to this study were selected from people who were referred to polysomnographic assessment at Ministry of Health Dışkapı Yıldırım Beyazıt Research and Educational Hospital, Sleep Laboratory, Ankara, Turkey. The 30 patients who had moderate-to-severe OSA (apnea-hypopnea index $[\mathrm{AHI}] \geq 15$ events/hour, 13 females, 17 males, mean age: $49 \pm 13$ years) and were planned to be treated with CPAP were enrolled as patient group and 18 individuals in whom the diagnosis of OSA was excluded (AHI $<5$ events/hour, 8 females, 10 males, mean age: $51 \pm$
4 years) were enrolled as control group. Patients who had history of cardiac arrhythmia, coronary artery disease, cardiomyopathies, valvular heart disease, pericardial disease, pulmonary emboli or pulmonary hypertension, atrioventricular or intraventricular conduction abnormalities, abnormal thyroid function, and patients who were receiving antiarrhythmic medications were excluded from the study. All study patients were in sinus rhythm. Pretherapy echocardiographic and electrocardiographic assessment of the control group and the patient group were performed. The patient group was trained about CPAP therapy and an appointment for reassessment was scheduled for after 6 months of the therapy. Effective CPAP therapy was determined to require patient administration for at least 5 hours a night and for at least 5 days a week. After 6 months, one of 30 patients could not be reached and 5 patients informed that for a variety of reasons they could not use the device. The echocardiographic and electrocardiographic assessments of the remaining 24 patients who had effective CPAP therapy (11 females, 12 males, average age $50 \pm 12$ ) were repeated. Written informed consent was obtained from all the patients that were included in this study. The local research ethics committee approved our study.

\section{Polysomnography}

All patients were subject to polysomnographic record using a whole night using a 64-channel Compumedics (Melbourne, Victoria, Australia) E-series polysomnography device. Sleep stages were detailed by standard electroencephalogaraphic, electrooculographic criteria. Apneas and hypnoeas were recorded by oronasal flow cannulae attached to pneumotachography. Arterial oxygen saturation was measured by pulse oximetry using a finger probe. The variables analyzed were total sleep time, sleep efficiency, sleep latency, rapid eye movement (REM) and non-REM sleep percentages, AHI (apnea + hypopnea events per hour), arousals/hour, and oxygen saturation. Apnea was defined as a decrease in airflow of at least $80 \%$ for 10 seconds or more, and hypopnea was defined as a decrease in airflow of at least $50 \%$ for 10 seconds or more. An AHI > 5 was considered diagnostic for OSA. The groups were defined as controls (AHI $\leq 5$ events/hour) and moderate-tosevere (AHI $\geq 15$ events/hour) OSA. 


\section{Echocardiographic Measurements}

Two-dimensional, M-mode pulsed and color flow Doppler echocardiographic examinations of all subjects were performed by the same experienced echocardiographer with same equipment /Vivid 7 pro, GE, Horten, Norway, 2-4 $\mathrm{mHz}$ phased array transducer). During echocardiographic examination, a single-lead electrocardiogram was recorded continuously. M-mode measurements were performed according to criteria of American Society of Echocardiography ${ }^{17}$ Left atrium (LA) diameter, left ventricle (LV) end-systolic and enddiastolic diameters were measured. LV ejection fraction (LVEF) was assessed by Simpson's rule. Pulsed-wave mitral flow velocities were measured from the apical four-chamber view by inserting a sample volume to mitral leaflet tips. Mitral early diastolic velocity $(\mathrm{E}, \mathrm{cm} / \mathrm{s})$, late diastolic velocity $(\mathrm{A}, \mathrm{cm} / \mathrm{s}), \mathrm{E} / \mathrm{A}$ ratio, deceleration time (DT), and isovolumic relaxation time (IVRT) were determined. Each representative value was obtained from the average of three measurements. The operator was blinded to the clinical details and results of the other investigations of each patient and controls. Doppler tissue imaging echocardiography was performed by transducer frequencies of $3.5-4.0 \mathrm{MHz}$, adjusting the spectral pulsed Doppler signal filters until a Nyquist limit of $15-20 \mathrm{~cm} / \mathrm{s}$ was reached, and using the minimal optimal gain. The monitor sweep speed was set at $50-100 \mathrm{~mm} / \mathrm{s}$ to optimize the spectral display of myocardial velocities. In an apical four-chamber view, the pulsed Doppler sample volume was placed at the level of LV lateral mitral annulus, septal mitral annulus, and right ventricular (RV) tricuspid annulus. The time interval from the onset of the $\mathrm{P}$ wave on surface ECG to the beginning of the late diastolic wave (Am), which is named PA, was obtained from the lateral mitral annulus (lateral PA), septal mitral annulus (septal $\mathrm{PA}$, and RV tricuspid annulus (tricuspid PA). The difference between lateral PA and tricuspid PA was defined as interatrial electromechanical delay (lateral PA - tricuspid PA). The difference between septal PA and tricuspid PA was defined as right-sided intraatrial electromechanical delay (septal PA - tricuspid PA). The difference between lateral PA and septal PA was defined as left-sided intraatrial electromechanical delay (lateral PA septal PA).

\section{Pd Measurements on 12-Lead ECGs}

All standard 12-lead ECGs were obtained simultaneously using a recorder (Hewlett Packard, Pagewriter, Andover, MA, USA) set at a $50 \mathrm{~mm} / \mathrm{s}$ paper speed and $2 \mathrm{mV} / \mathrm{cm}$ standardization. A single cardiologist, who was blinded to the clinical status of the patients, measured ECG intervals. To decrease the error measurements, P-wave duration was measured manually with calibers and magnifying glass. The onset of the $\mathrm{P}$ wave was defined as the point of first visible upward slope from baseline for positive waveforms and as the point of first downward slope from baseline for negative waveforms. Return to the baseline was considered as the end of the $\mathrm{P}$ wave. Pmax measured in any of the 12 leads of the surface ECG was used as the longest atrial conduction time and $\mathrm{P}$ minimum (Pmin) measured in any of the 12 leads of the surface ECG was used as the shortest atrial conduction time. The difference between the Pmax and the Pmin was calculated and defined as Pd.

\section{Statistical Analysis}

SPSS 17.0 for Windows statistical package program was used for statistical analysis (IBM Corporation; Somers, NY, USA). All data were expressed as mean \pm SD and frequency (\%). Student's $t$-test was used for the comparison of continuous variables. Paired $t$-test was performed to compare baseline and posttreatment electrocardiographic and echocardiographic parameters in patients with OSA. The relationship between the parameters was evaluated with the Pearson coefficient of correlation. A P value of $<0.05$ was considered statistically significant.

\section{RESULTS}

Clinical and laboratory characteristics of the study groups are shown in Table 1 . There was no significant difference between patients with OSA and controls with respect to age, sex, body mass index, smoking rate, presence of diabetes mellitus, and hypertension. As expected, AHI was significantly higher and the lowest oxygen saturation was significantly lower in patients with OSA as compared to controls. Pmax and Pd were significantly higher in the OSA group as compared to controls. Regarding echocardiographic 
Table 1. Clinical, Laboratory, Conventional and Tissue Doppler Echocardiographic and Electrocardiographic Characteristics of Study Population

\begin{tabular}{|c|c|c|c|}
\hline & $\begin{array}{c}\text { OSA } \\
(n=24)\end{array}$ & $\begin{array}{l}\text { Control } \\
(n=18)\end{array}$ & P Value \\
\hline Age (years) & $50 \pm 12$ & $51 \pm 4$ & 0.8 \\
\hline Female, n (\%) & $11(46)$ & $8(44)$ & 0.9 \\
\hline BMI $\left(\mathrm{kg} / \mathrm{m}^{2}\right)$ & $34 \pm 7$ & $31 \pm 3$ & 0.07 \\
\hline Diabetes mellitus, n (\%) & $4(17)$ & $2(11)$ & 0.6 \\
\hline Hypertension, n (\%) & 8 (33) & $6(33)$ & 1 \\
\hline Hyperlipidemia, n (\%) & $13(54)$ & 7 (39) & 0.3 \\
\hline Smokers, n (\%) & $9(37)$ & $5(28)$ & 0.5 \\
\hline $\mathrm{AHI}$ (events/hour) & $48 \pm 27$ & $2.2 \pm 0.8$ & $<0.0001$ \\
\hline Lowest oxygen saturation (\%) & $76 \pm 10$ & $87 \pm 4$ & $<0.0001$ \\
\hline \multicolumn{4}{|c|}{$\begin{array}{l}\text { Conventional Echocardiographic } \\
\text { Parameters }\end{array}$} \\
\hline LVEF (\%) & $59.0 \pm 4.0$ & $61.0 \pm 2.0$ & 0.2 \\
\hline LVEF (\%) & $59.0 \pm 4.0$ & $61.0 \pm 2.0$ & 0.2 \\
\hline $\mathrm{PW}(\mathrm{cm})$ & $0.96 \pm 0.30$ & $1.0 \pm 0.10$ & 0.6 \\
\hline IVS (cm) & $1.05 \pm 0.10$ & $1.30 \pm 0.10$ & 0.5 \\
\hline LA dimension $(\mathrm{cm})$ & $3.70 \pm 0.30$ & $3.20 \pm 0.20$ & $<0.0001$ \\
\hline $\mathrm{RA}$ area $\left(\mathrm{cm}^{2}\right)$ & $13.9 \pm 5.0$ & $9.50 \pm 1.60$ & 0.004 \\
\hline$E(\mathrm{~m} / \mathrm{s})$ & $0.67 \pm 0.12$ & $0.85 \pm 0.20$ & $<0.0001$ \\
\hline $\mathrm{A}(\mathrm{m} / \mathrm{s})$ & $0.77 \pm 0.14$ & $0.74 \pm 0.10$ & 0.6 \\
\hline$E / A$ & $0.90 \pm 0.10$ & $1.20 \pm 0.20$ & $<0.0001$ \\
\hline DT (ms) & $200 \pm 47$ & $194 \pm 31$ & 0.6 \\
\hline IVRT (ms) & $129 \pm 26$ & $114 \pm 11$ & 0.03 \\
\hline \multicolumn{4}{|l|}{ Tissue Doppler Parameters } \\
\hline Lateral PA (ms) & $84.5 \pm 9.2$ & $61.6 \pm 4.4$ & $<0.0001$ \\
\hline Septal PA (ms) & $64.0 \pm 12.0$ & $50.5 \pm 4.0$ & $<0.0001$ \\
\hline Tricuspid PA (ms) & $44.3 \pm 5.0$ & $40.5 \pm 2.0$ & $<0.0001$ \\
\hline Interatrial EMD (ms) & $39.2 \pm 8.0$ & $21.1 \pm 2.8$ & $<0.0001$ \\
\hline Left-sided intraatrial (ms) & $20.5 \pm 7.2$ & $11.1 \pm 2.0$ & 0.003 \\
\hline Right-sided intraatrial (ms) & $20.7 \pm 11.0$ & $10.0 \pm 2.6$ & $<0.0001$ \\
\hline \multicolumn{4}{|c|}{ Electrocardiographic Parameters } \\
\hline Heart rate (beats/min) & $78 \pm 8$ & $79 \pm 9$ & 0.6 \\
\hline $\mathrm{P}$ maximum $(\mathrm{ms})$ & $130.0 \pm 9.0$ & $112.0 \pm 10.0$ & $<0.0001$ \\
\hline P minimum (ms) & $86.1 \pm 6.0$ & $82.3 \pm 0.7$ & 0.1 \\
\hline P-wave dispersion (ms) & $44.0 \pm 7.0$ & $28.5 \pm 4.0$ & $<0.0001$ \\
\hline
\end{tabular}

$\mathrm{AHI}=$ apnea-hypopnea index; $\mathrm{BMI}=$ body mass index; DT = deceleration time; EMD = electromechanical delay; IVRT = isovolumic relaxation time; IVS = interventricular septum thickness; LA = left atrium; LVEF = left ventricular ejection time; PW = posterior wall thickness; RA = right atrium. Bold values are statistically significant $(p \leq 0.05)$.

comparisons, there was no significant difference in LVEF between groups. However, E-wave velocity of mitral inflow and E/A ratio were significantly lower and IVRT, LA diameter, and right atrial (RA) area were significantly higher in the OSA group as compared to those without OSA. In addition, lateral $\mathrm{PA}$, septalt $\mathrm{PA}$, and tricuspid PA were significantly higher in OSA group than those without OSA. Moreover, interatrial, left-sided intraatrial, and right-sided intraatrial electromechanical delays were significantly higher in OSA group as compared with the controls (Table 1). In the OSA group, there were significant positive correlations between AHI and Pmax $(\mathrm{r}=00.8, \mathrm{P}>0.0001), \mathrm{Pd}(\mathrm{r}=0.9, \mathrm{P}<0.0001)$, interatrial electromechanical delay $(\mathrm{r}=0.8$, $\mathrm{P}<0.0001$ ) and intraatrial electromechanical delay both left and right side $(\mathrm{r}=0.4, \mathrm{P}=0.04$ and $\mathrm{r}=0.6, \mathrm{P}<0.0001$, respectively). However, significant negative correlation was noted between lowest oxygen saturation and $P \max (\mathrm{r}=-0.4$, $\mathrm{P}<0.003), \mathrm{Pd}(\mathrm{r}=-0.5, \mathrm{P}<0.0001)$, interatrial electromechanical delay $(\mathrm{r}=-0.5, \mathrm{P}<0.0001)$, and intraatrial electromechanical delays both left and right side $(\mathrm{r}=-0.3, \mathrm{P}=0.003$ and $\mathrm{r}=-0.6$, $\mathrm{P}<0.0001$, respectively). 

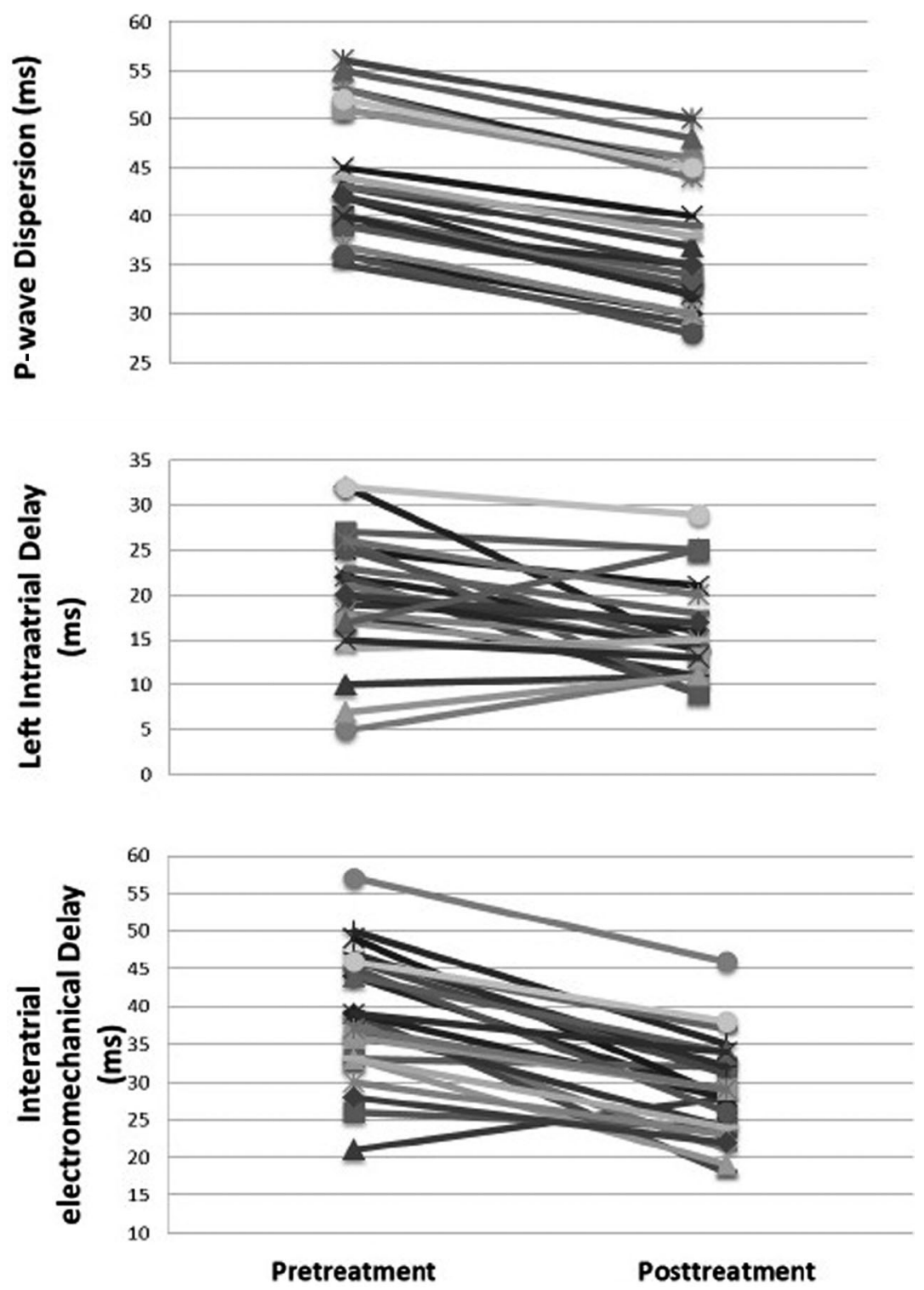

Figure 1. Pre- and posttreatment comparisons of P-wave dispersion, left intraatrial electromechanical delay, and interatrial electromechanical delay in patients with obstructive sleep apnea.

\section{Impact of CPAP Therapy on Electrocardiographic and Echocardiographic Parameters}

Electrocardiographic and echocardiographic parameters were reevaluated after 6 months of CPAP therapy in patients with OSA (Fig. 1, Table 2). Significant reduction was noted in lateral PA, septal PA, and tricuspid PA values after CPAP therapy as compared to baseline values. Also, significant reduction was present in the interatrial, left-sided intraatrial, and right-sided intraatrial electromechanical delays. Moreover, both Pmax and $\mathrm{Pd}$ values decreased significantly after CPAP therapy as compared to baseline. In addition to those, indicators of diastolic function such as $\mathrm{E} / \mathrm{A}$ rate and IVRT improved significantly after treatment of CPAP as compared to pretreatment values. Also LA diameter decreased significantly after CPAP therapy as compared to baseline values. However, no significant change was noted before and after treatment in RA area in patients with OSA. 
Table 2. Impact of Continuous Positive Airway Pressure Treatment on Echocardiographic and Electrocardiographic Parameters

\begin{tabular}{lccc}
\hline & Baseline & Posttreatment & P Value \\
\hline Heart rate (beats/min) & $78 \pm 8$ & $79 \pm 11$ & 0.6 \\
Lateral PA (ms) & $84.5 \pm 9.2$ & $70.3 \pm 6.8$ & $<0.001$ \\
Septal PA (ms) & $64.0 \pm 12.0$ & $54.7 \pm 7.7$ & $<0.0001$ \\
Tricuspid PA (ms) & $44.3 \pm 5.0$ & $41.6 \pm 4.3$ & $\mathbf{0 . 0 2}$ \\
Interatrial EMD (ms) & $39.2 \pm 8.0$ & $28.7 \pm 6.5$ & $<.0001$ \\
Left-sided intraatrial delay (ms) & $20.5 \pm 7.2$ & $15.6 \pm 5.1$ & $\mathbf{0 . 0 0 2}$ \\
Right-sided intraatrial delay (ms) & $20.7 \pm 11.0$ & $13.1 \pm 7.3$ & $<0.0001$ \\
LA dimension (cm) & $3.70 \pm 0.30$ & $13.5 \pm 0.20$ & $<.0001$ \\
RA area (cm ${ }^{2}$ ) & $13.9 \pm 5.0$ & $0.81 \pm 0.11$ & 0.6 \\
E (m/s) & $0.67 \pm 0.12$ & $0.76 \pm 0.13$ & 0.7 \\
A (m/s) & $0.77 \pm 0.14$ & $1.10 \pm 0.20$ & $<0.0001$ \\
E/A & $0.90 \pm 0.10$ & $124.0 \pm 11.0$ & $<0.0001$ \\
P maximum (ms) & $130.0 \pm 9.0$ & $87.0 \pm 7.0$ & 0.08 \\
P minimum (ms) & $86.1 \pm 6.0$ & $37.0 \pm 7.0$ & $<0.0001$ \\
P-wave dispersion (ms) & $44.0 \pm 7.0$ & &
\end{tabular}

Abbreviations as in Table 1. Bold values are statistically significant $(p \leq 0.05)$.

\section{DISCUSSION}

In this study, we have shown that patients with OSA had significantly longer interatrial electromechanical delay, left-sided electromechanical delay, and right-sided intraatrial electromechanical delay as compared to subjects without OSA. Also, some electrocardiographic markers which are thought to be related to increased $\mathrm{AF}$ risk including Pmax and $\mathrm{Pd}$ were found to be significantly higher in patients with OSA as compared to controls. However, 6-month duration of CPAP therapy resulted in significant improvements in these TDI echocardiographic and electrocardiographic parameters in patients with OSA. Besides, we found CPAP therapy had beneficial effects on diastolic function of $\mathrm{LV}$ in OSA patients. According to our knowledge, this is the first study which shows significant beneficial effects of CPAP therapy on AF risk markers in OSA patients.

Previous studies have shown higher incidence of brady- and tachyarrhythmia in patients with OSA in relation with the severity of the disease and the level of hypoxia. ${ }^{18,19} \mathrm{~A}$ strong association has also been shown between OSA and AF. ${ }^{20}$ Previous studies have demonstrated that prolonged intra- and interatrial conduction times and inhomogeneous propagation of sinus impulses would be the characteristics of increased $\mathrm{AF}$ risk in various clinical conditions during sinus rhythm. ${ }^{7}$ Also, increased electromechanical delays in both atria and greater $\mathrm{Pd}$ have been shown in patients with OSA. ${ }^{13,14}$ Our results are in accordance with the previous findings. Although the underlying mechanism for the higher incidence of $\mathrm{AF}$ in patients with OSA is not fully clear, hypoxia is thought to be the essential mechanism for increased AF risk. ${ }^{21}$ Accordingly, lowest oxygen saturation was found to be in the OSA group in our study. Besides, there was a moderate but significant negative correlation between the lowest oxygen saturation and echocardiographic as well as electrocardiographic markers of increased AF risk in our study. The other probable mechanisms involved in predisposition to AF in OSA are structural change in cardiac chamber size, LV diastolic dysfunction, increased negative intrathoracic pressure, systemic inflammation, and higher sympathetic activation. ${ }^{22,23}$ In our basal conventional echocardiographic assessment prior to the CPAP treatment, we found significant differences in parameters that are the indicators of diastolic dysfunction (E/A ratio, IVRT) and in LA dimension in OSA group as compared to the control group.

CPAP therapy is the common and essential treatment of OSA and several studies have shown significant reduction in the cardiovascular morbidity and mortality after CPAP treatment in patients with OSA. ${ }^{24,25}$ Nasal CPAP therapy ensures decrease in increased negative intrathoracic pressure and reduction in apneic-hypopneic incidents. ${ }^{26}$ These effects of CPAP therapy has been shown to be resulted in decrease in LA volume and LA volume index. ${ }^{27}$ In addition, improvement 
in both the systolic and diastolic functions of LV has been demonstrated after effective CPAP therapy in patients with OSA. ${ }^{28,29}$ Hypoxia due to apnea episodes in patients with OSA may also cause autonomic imbalance which may facilitate the development of AF. Therefore, frequency of ventricular and supraventricular tachycardia associated with OSA can be mitigated by CPAP therapy which reduces the number of apnea periods and intermittent hypoxia. ${ }^{30}$ In a study by Kanagala et al. conducted in patients with OSA who underwent successful cardioversion for $\mathrm{AF}$, recurrence rate of $\mathrm{AF}$ was $82 \%$ within 1 year in patients with OSA who did not receive diseasespecific therapy and this was the twice of the group that effectively received therapy. ${ }^{31}$ Also, after AF catheter ablation $25 \%$ greater risk of $\mathrm{AF}$ recurrence has been determined in OSA patients than those without OSA. ${ }^{32}$ All these findings suggested that effective CPAP therapy might result in decreased AF rate by mainly decreasing the apnea episodes. As shown in our study, significant improvements in LV diastolic function as well as more homogenous conduction through the atria might contribute the decreased AF rate in OSA patients after effective CPAP therapy.

In addition to small sample size, there are some limitations that must be taken into account in our study. First, there were no patients with documented $\mathrm{AF}$ in both groups. In previous studies however, investigated parameters have all been shown to be related to increased AF risk in various cardiovascular conditions. Therefore, we think documentation of improvement in these probable $\mathrm{AF}$ risk markers after effective CPAP therapy is invaluable. Second, only CPAP therapy of OSA was assessed in our study. Surgical correction of obstruction in upper airways might reveal greater and permanent improvements in such patients. Studies are needed to evaluate the impact of surgical treatment particularly on cardiovascular outcomes in patients with OSA.

\section{CONCLUSION}

In conclusion, our findings suggested that increased inter- and intraatrial electromechanic delays and prolonged Pmax and Pd are evident in patients with OSA and improvement in these AF risk markers as well as in diastolic LV function could be obtained after effective CPAP therapy.
Further studies with larger patient population are needed to evaluate the effects of CPAP and surgical therapy of OSA on cardiovascular outcomes.

\section{REFERENCES}

1. Kannel WB, Wolf PA, Benjamin EJ, et al. Prevalence, incidence, prognosis and predisposing condition for atrial fibrillation: Population-based estimates. Am J Cardiol $1998 ; 82(8 \mathrm{~A}): 2 \mathrm{~N}-9 \mathrm{~N}$.

2. Lattimore JD, Celermajer DS, Wilcox I. Obstructive sleep apnea and cardiovascular disease. J Am Coll Cardiol 2003;41:1429-1437.

3. Caples SM, Gami AS, Somers VK. Obstructive sleep apnea. Ann Intern Med 2005;142:187-197.

4. Go AS, Hylek EM, Phillips KA, et al. Prevalence of diagnosed atrial fibrillation in adults: National implications for rhythm management and stroke prevention: The Anticoagulation and Risk Factors in Atrial Fibrillation (ATRIA) study. JAMA 2001;285:2370-2375.

5. Benjamin EJ, Wolf PA, D'Agostino RB, et al. Impact of atrial fibrillation on the risk of death: The Framingham Heart Study. Circulation 1998;98:946-952.

6. Gami AS, Pressman G, Caples SM, et al. Association of atrial fibrillation and obstructive sleep apnea. Circulation 2004;110(4):364-367.

7. Daubert JC, Pavin D, Jauvert G, et al. Intra- and interatrial conduction delay: Implications for cardiac pacing. Pacing Clin Electrophysiol 2004;27(4):507-525.

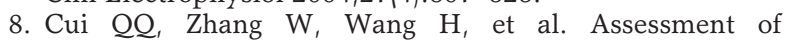
atrial electromechanical coupling and influential factors in nonrheumatic paroxysmal atrial fibrillation. Clin Cardiol 2008;31:74-78.

9. Köse S, Aytemir K, Sade E, et al. Detection of patients with hypertrophic cardiomyopathy at risk for paroxysmal atrial fibrillation during sinus rhythm by P-wave dispersion. Clin Cardiol 2003;26(9):431-434.

10. Kannel WB, Abbott RD, Savage DD, et al. Epidemiologic features of chronic atrial fi-brillation: The Framingham study. N Engl J Med 1982;306:1018-1022.

11. Demirkan B, Guray Y, Guray U, et al. The acute effect of percutaneous mitral balloon valvuloplasty on atrial electromechanical delay and $\mathrm{P}$-wave dispersion in patients with mitral stenosis. Herz 2013;38(2):210-215.

12. Guray U, Guray Y, Mecit B, et al. Maximum p wave duration and $\mathrm{p}$ wave dispersion in adult patients with secundum atrial septal defect: The impact of surgical repair. Ann Noninvasive Electrocardiol 2004;9(2):136-141.

13. Yagmur J, Yetkin O, Cansel M, et al. Assessment of atrial electromechanical delay and influential factors in patients with obstructive sleep apnea. Sleep Breath 2012;16(1):8388.

14. Cagirci G, Cay S, Gulsoy KG, et al. Tissue Doppler atrial conduction times and electrocardiogram interlead P-wave durations with varying severity of obstructive sleep apnea. J Electrocardiol 2011;44(4):478-482.

15. Sullivan C, Issa FG, Berthon-Jones, et al. Reversal of obstructive sleep apnea by continuous positive airway pressure applied through the nares. Lancet 1981;1(8225):862865.

16. Waldhorn RE, Herrick TW, Nguyen MC, et al. Long-term compliance with nasal continuous positive airway pressure therapy of obstructive sleep apnea. Chest 1990;97(1):33-38.

17. Parish JM, Somers VK. Obstructive sleep apnea and cardiovascular disease. Mayo Clin Proc 2004;79(8):10361046. 
18. Guilleminault C, Connolly SJ, Winkle RA. Cardiac arrhythmia and conduction disturbances during sleep in 400 patients with sleep apnea syndrome. Am J Cardiol 1983;52(5):490-494.

19. Shepard JW, Jr, Garrison MW, Grither DA, et al. Relationship of ventricular ectopy to oxyhemoglobin desaturation in patients with obstructive sleep apnea. Chest 1985:88(3):335-340.

20. Gami AS, Hodge DO, Herges RM, et al. Obstructive sleep apnea, obesity, and the risk of incident atrial fibrillation. J Am Coll Cardiol 2007;49(5):565-571.

21. Visser M, Bouter LM, McQuillan GM, et al. Elevated Creactive protein levels in overweight and obese adults. JAMA 1999;282(22):2131-2135.

22. Niroumand $M$, Kuperstein $R$, Sasson $Z$, et al. Impact of obstructive sleep apnea on left ventricular mass and diastolic function. Am J Respir Crit Care Med 2001;163:1632-1636.

23. Kraiczi H, Caidahl $\mathrm{K}$, Samuelsson $A$, et al. Impairment of vascular endothelial function and left ventricular filling: Association with the severity of apnea-induced hypoxemia during sleep. Chest 2001;119: 1085-1091.

24. Marin JM, Carrizo SJ, Vicente E, et al. Long-term cardiovascular outcomes in men with obstructive sleep apnoeahypopnoea with or without treatment with continuous positive airway pressure: An observational study. Lancet 2005;365:1046-1053.
25. Campos-Rodriguez F, Pena-Grinan N, Reyes-Nunez N, et al. Mortality in obstructive sleep apnea-hypopnea patients treated with positive airway pressure. Chest 2005; 128:624-633.

26. Dhillon $\mathrm{S}$, Chung SA, Fargher $\mathrm{T}$, et al. Sleep apnea, hypertension, and the effects of continuous positive airway pressure. Am J Hypertens 2005; 18:594-600.

27. Khan A, Latif F, Hawkins B, et al. Effects of obstructive sleep apnea treatment on left atrial volume and left atrial volume index. Sleep Breath 2008;12(2):141-147.

28. Bradley TD, Logan AG, Kimoff $R J$, et al; CANPAP Investigators. Continuous positive airway pressure for central sleep apnea and heart failure. $\mathrm{N}$ Engl J Med 2005;353(19):2025-2033.

29. Arias MA, Garcia-Rio F, Alonso-Fernandez A, et al. Obstructive sleep apnea syndrome affects left ventricular diastolic function: Effects of nasal continuous positive airway pressure in men. Circulation 2005;112:375-383.

30. Harbison J, O'Reilly P, McNicholas WT. Cardiac rhythm disturbances in the obstructive sleep apnea syndrome: Effects of nasal continuous positive airway pressure therapy. Chest 2000;118:591-595.

31. Kanagala R, Murali RS, Friedman PA, et al. Obstructive sleep apnea and the recurrence of atrial fibrillation. Circulation 2003;107:2589-2594.

32. Ng CY, Liu T, Shehata M, et al. Meta-analysis of obstructive sleep apnea as predictor of atrial fibrillation recurrence after catheter ablation. Am J Cardiol 2011;108(1):47-51. 